\title{
CONTROLE QUÍMICO EM PÓS-EMERGÊNCIA DE CAPIM-GENGIBRE (Paspalum maritimum Trin.)
}

\author{
Antônio José Plácido de Mello ${ }^{1 *}$, Renan Cantalice de Souza², Siumar Pedro Tironi ${ }^{3}$ \\ ${ }^{1}$ IFAL Campus Palmeira dos Índios - Alagoas, Avenida Palmeira de Fora, s/n, Palmeira dos Índios, AL, CEP 57601-220. \\ 2UFAL Centro de Ciências Agrárias, BR 104 km 35,s/n, Rio Largo, AL, CEP 57100-000 \\ 3UFFS Avenida Fernando Machado, 180, Centro, Chapecó, SC, CEP 89802-112. \\ *Autor para correspondência: Antônio José Plácido de Mello, placido@ifalpalmeira.edu.br
}

\begin{abstract}
RESUMO: Capim-gengibre planta daninha de difícil controle e ocorrência regional. Objetivou-se avaliar a eficiência de herbicidas no controle em pós-emergência do capim-gengibre no município de Rio Largo, Estado de Alagoas. Instalado em blocos casualizados em parcelas subdivididas no tempo, com quatro repetições, cada unidade experimental foi composta de $3 \mathrm{~m}$ de largura por $6 \mathrm{~m}$ de comprimento. Os tratamentos foram compostos por herbicidas, aplicados isoladamente ou em associações, utilizados em pós-emergência tardia. Os herbicidas utilizados nos tratamentos foram: T1 - metribuzin (200 g i.a ha-1); T2 - metsulfuron-methyl (22 $\mathrm{g}$ i.a ha-1); T3 - glyphosate (1800 g i.a ha-1 e a.e. $\left.1350 \mathrm{~g} \mathrm{~L}^{-1}\right)$; T4 - paraquat + diuron (400 g i.a ha-1 $+100 \mathrm{~g}$ i.a ha-1); T5 imazapic (123 g i.a ha-1); T6 - MSMA + diuron (200 g i.a ha-1 + $100 \mathrm{~g}$ i.a ha-1); T7 - Testemunha (sem aplicação de herbicida). A aplicação dos herbicidas foi realizada sobre a espécie daninha e palhada da cultura, aos sete dias após o corte mecanizado da cana-de-açúcar. A variável observada foi percentual de controle visual do capimgengibre. Os resultados permitiram concluir que existe diferença no controle com MSMA + diuron, paraquat + diuron, metsulfuron methyl e glyphosate.
\end{abstract}

PALAVRAS-CHAVES: Competição, Cana-de-açúcar e metsulfuron methyl.

\section{CHEMICAL CONTROL IN POST EMERGENCE OF GINGER GRASS (Paspalum maritimum TRIN.)}

ABSTRACT: Grass ginger weed unwieldy and regional occurrence.This study aimed to evaluate the efficiency of herbicides in controlling post-emergence of grass-ginger in Rio Largo municipality, State of Alagoas. Installed in a randomized block in split plot, with four replications, each experimental unit consisted of $3 \mathrm{~m}$ wide and $6 \mathrm{~m}$ long. The treatments consisted of herbicides, applied alone or in associations, used in late post emergence. Herbicides used in the treatments were: T1 - metribuzin ( $\left.200 \mathrm{~g}^{\text {i.a ha }}{ }^{-1}\right)$; T2 - metsulfuron-methyl (22 $\mathrm{g}$ i.a ha-1 $)$; T3 - glyphosate (1800 $\mathrm{g}_{\text {i.a ha }}{ }^{-1}$ and a.e. $\left.1350 \mathrm{~g} \mathrm{~L}^{-1}\right)$; T4 - paraquat + diuron (400 $\mathrm{g}_{\text {i.a ha }}{ }^{-1}+100 \mathrm{~g}^{-1 . a}$ ha $\left.{ }^{-1}\right) ; \mathrm{T} 5$ - imazapic (123 g

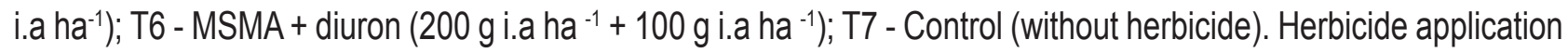
was performed on the weed and trash culture, the seventh day after the mechanical harvesting of sugarcane. The observed variable was the percentage of visual control of grass ginger. The results showed that there is difference in control with MSMA + diuron, paraquat + diuron, metsulfuron methyl and glyphosate.

KEYWORDS: Competition, cane sugar and methyl metsulfuron.

\section{INTRODUÇÃO}

0 capim-gengibre (Paspalum maritimum Trin.) é uma planta daninha de ocorrência regional, abrangendo os canaviais dos Estados da Paraíba, Pernambuco, Alagoas e Sergipe. Quando não controlada forma reboleiras extensas, especialmente em solos de baixa fertilidade e/ou arenosos (Maciel et al., 2010). A prática de manutenção da vegetação natural em algumas culturas através da roçagem mecânica favorece o aumento da incidência de capim- gengibre em detrimento de outras espécies pela sua agressividade e o desenvolvimento vegetativo (Fontes, 2014). Para Oliveira et al. (2013) trata-se de uma das plantas daninhas mais importantes da Região Nordeste, infestando principalmente lavouras perenes, culturas anuais e beiras de estradas. Devido à sua biologia de reprodução sexuada e assexuada, através de semente, estolão e rizomas são de difícil controle, aliado a sua alta capacidade de produção de biomassa, capacidade de adaptação aos mais variados ambientes. Além 
disso, sua importância está relacionada com o efeito alelopático em relação à cana-de-açúcar e com outras plantas daninhas. Estas situações citadas dificultam 0 controle do capim-gengibre nos canaviais nordestino. Os produtos existentes controlam por período curto permitindo sua rebrota e/ou causam injurias à cultura. 0 presente trabalho teve como objetivo avaliar a eficiência de herbicidas no controle em pós-emergência do capim gengibre em campo.

\section{MATERIAL E MÉTODOS}

O ensaio foi conduzido no município de Rio Largo/AL (Latitude de $9^{\circ} 27^{\prime} \mathrm{S}$; Longitude de $35^{\circ} 27^{\prime}$ W e Altitude de $127 \mathrm{~m}$ ), no período de abril a junho de 2013, na Fazenda Cuia Velha pertencente à Usina Santa Clotilde, em área de produção de cana-de-açúcar, com colheita mecanizada e irrigada. Foi instalado em blocos casualizados com parcelas subdivididas no tempo, com quatro repetições, sendo cada unidade experimental composta de 3,0 m largura por 6,0 m de comprimento. Desse modo, a área total de cada parcela foi de $18 \mathrm{~m}^{2}$ e utilizou-se a cultivar RB 92579 na condição de canasoca, espaçadas com $1,4 \mathrm{~m}$ entre linhas, $6^{\circ}$ folha. A área experimental foi na densidade de $15 \mathrm{t} \mathrm{ha}^{-1} \mathrm{de}$ palha.

Para a determinação da densidade foi retirada a palhada em 5 pontos ao acaso com 0 quadrado de $0,5 \times 0,5 \mathrm{~m}$ e 0 material pesado em laboratório, em uma mancha de infestação de $P$. maritimum, capim-gengibre. Os tratos culturais foram realizados conforme recomendação para cultura. A aplicação dos tratamentos foi realizada utilizando-se um pulverizador costal pressurizado a $\mathrm{CO}_{2}$, munido de bicos tipo leque (Teejet TTI 110 02-VS), mantido à pressão constante, proporcionando volume de calda de $200 \mathrm{~L} \mathrm{ha}^{-1}$, a barra de pulverização foi mantida a $0,5 \mathrm{~m}$ de altura do alvo.
Durante a aplicação a temperatura do ar estava com $27,7^{\circ} \mathrm{C}$, umidade relativa do ar $67 \%$, velocidade do vento $3,4 \mathrm{~m} \mathrm{~s}^{-1}$ e temperatura do solo a $10 \mathrm{~cm}$ de profundidade $32,9^{\circ} \mathrm{C}$.

Os tratamentos foram compostos por herbicidas, aplicados isoladamente ou em associações, utilizados em pós-emergência tardia. Os herbicidas utilizados nos tratamentos foram: T1 - metribuzin (200 g i.a ha-1); T2 metsulfuron-methyl (22 g i.a ha-1); T3 - glyphosate (1800 g i.a ha-1 e $1350 \mathrm{~g} \mathrm{~L}^{-1}$ equivalente ácido); T4 - paraquat + diuron (400 g i.a ha-1 $+100 \mathrm{~g}$ i.a ha-1); T5 - imazapic (123 g i.a ha-1); T6 - MSMA + diuron (200 g i.a ha-1 + 100 g i.a ha-1); 77 - Testemunha (sem aplicação de herbicida).

A aplicação dos herbicidas foi realizada sobre a espécie daninha e palhada da cultura, aos trinta dias após o corte mecanizado da cana-de-açúcar. A variável observada foi percentual de controle visual do capimgengibre (\%). Após a aplicação dos herbicidas foram realizadas avaliação visual de controle aos $7,14,21$, $28,35,42,49,56,63$ dias após a aplicação (DAA), em que 0 representa ausência total de sintomas e todas as plantas vivas e $100 \%$ a morte da planta. Os dados foram submetidos à análise de variância utilizando o software SISVAR e quando constatada a diferença significativa, foram comparados pelo teste de Tukey, $p<0,05$ de probabilidade de erro. A percentagem de controle visual do capim-gengibre foi submetida à determinação do modelo da equação de regressão escolhendo a equação pelo maior valor do coeficiente de determinação - $R^{2}$.

\section{RESULTADOS E DISCUSSÃO}

Observou-se que a diferença é significativa pelo teste $\mathrm{F}$ a $1 \%$ de probabilidade para Época de avaliação $(A)$, Herbicidas (B) e para interação entre os fatores (AXB) encontram-se na Tabela 1.

Tabela 1. Resumo do quadro da análise de variância para a porcentagem de controle visual do capim-gengibre submetido ao controle químico, ensaio de campo, Rio Largo - AL, 2013.

\begin{tabular}{lllll}
\hline Causa de Variação & GL & SQ & QM & F \\
\hline Blocos & 3 & 2310,3 & 770,1 & $10,0^{* *}$ \\
Época de Avaliação (A) & 8 & 27189,0 & 3398,6 & $44,3^{*}$ \\
Resíduo (a) & 24 & 1840,2 & 76,7 & $33,6^{*}$ \\
Herbicidas (B) & 6 & 85348,3 & 14224,7 & $3,6^{*}$ \\
Interação A X B & 48 & 73432,3 & 1529,8 & \\
Resíduo (b) & 162 & 68486,9 & 422,7 & \\
\hline Total & 251 & 258607,1 & & \\
\hline CV (a) & $25,78 \%$ & & & \\
\hline CV (b) & $60,53 \%$ & & \\
\hline GL - Graus de liberdade; SQ - Soma de quadrados; QM - Quadrado médio e F - valor calculado do teste F \\
** Diferença significativa a 1 \%
\end{tabular}


$\mathrm{Na}$ Tabela 2 observou-se diferenças de comportamento de tratamentos com relação ao controle de capim-gengibre quanto aos dias após a aplicação (DAA) pelo Teste de Tukey a $5 \%$ de probabilidade, 0 tratamento paraquat + diuron apresentou melhor performance aos 7 e 21 dias após aplicação (DAA); isso deve-se ao paraquat produto inibidor de fotossistema I com rápida absorção foliar, agindo em contato, a morte das plantas ocorre pelas perdas de fotossíntese dos tecidos afetados pela destruição dos ácidos graxos nos tilacoides e outras membranas celulares próximo ao locais de produção de radicais livres (Martins, 2013). Em poucas horas após a aplicação na presença de luz, verificou-se severa injúria nas folhas das plantas tratadas (Silva et al., 2014). Associado ao diuron inibidor da síntese de Hill, inibindo a evolução do oxigênio a partir da água na presença de cloroplastos e de um aceptor adequado de elétrons (Balke, 1985). Aos 28 a 63 dias após aplicação (DAA) o resultado do tratamento foi semelhante à testemunha.

Tabela 2. Média percentual de controle visual do capim-gengibre em função dos herbicidas e a época de avaliação (dias após a aplicação - DAA), Rio Largo - AL, 2013.

\begin{tabular}{llllllllll}
\hline Tratamentos & 07 & 14 & 21 & 28 & 35 & 42 & 49 & 56 & 63 \\
\hline metribuzin & $25,9 \mathrm{~b}$ & $28,1 \mathrm{~b}$ & $28,1 \mathrm{~b}$ & $32 \mathrm{~b}$ & $30,7 \mathrm{~b}$ & $30 \mathrm{~b}$ & $30 \mathrm{~b}$ & $30 \mathrm{~b}$ & $30 \mathrm{bc}$ \\
met. methyl & $46,5 \mathrm{c}$ & $52,1 \mathrm{c}$ & $52,2 \mathrm{c}$ & $64,6 \mathrm{c}$ & $70 \mathrm{c}$ & $68,8 \mathrm{c}$ & $65 \mathrm{c}$ & $61,3 \mathrm{~d}$ & $61,3 \mathrm{~d}$ \\
glyphosate & $48,1 \mathrm{c}$ & $49,4 \mathrm{c}$ & $49,4 \mathrm{c}$ & $70 \mathrm{c}$ & $62,5 \mathrm{c}$ & $58,8 \mathrm{c}$ & $55 \mathrm{c}$ & $42,5 \mathrm{c}$ & $42,5 \mathrm{c}$ \\
paraquat + diuron & $85,8 \mathrm{~d}$ & $52,1 \mathrm{c}$ & $86 \mathrm{~d}$ & $6,3 \mathrm{a}$ & $6,5 \mathrm{a}$ & $6,3 \mathrm{a}$ & $6,3 \mathrm{a}$ & $6,3 \mathrm{a}$ & $6,3 \mathrm{a}$ \\
imazapic & $8,8 \mathrm{bc}$ & $40,6 \mathrm{bc}$ & $40,6 \mathrm{bc}$ & $12,5 \mathrm{a}$ & $12,5 \mathrm{a}$ & $12,5 \mathrm{ab}$ & $11,3 \mathrm{a}$ & $11,3 \mathrm{a}$ & $11,3 \mathrm{a}$ \\
MSMA + diuron & $80,8 \mathrm{~d}$ & $88,8 \mathrm{~d}$ & $88,8 \mathrm{~d}$ & $18,8 \mathrm{ab}$ & $18,8 \mathrm{ab}$ & $16,3 \mathrm{ab}$ & $16,3 \mathrm{ab}$ & $15 \mathrm{a}$ & $16,5 \mathrm{ab}$ \\
testemunha & $0 \mathrm{a}$ & $0 \mathrm{a}$ & $0 \mathrm{a}$ & $0 \mathrm{a}$ & $0 \mathrm{a}$ & $0 \mathrm{a}$ & $0 \mathrm{a}$ & $0 \mathrm{a}$ & $0 \mathrm{a}$ \\
\hline
\end{tabular}

Médias seguidas da mesma letra na mesma coluna, não diferem pelo teste de Tukey a $5 \%$ de probabilidade.

O tratamento glyphosate aos 28 e aos 49 dias também apresentou diferença significativa pelo Teste de Tukey a $5 \%$ probabilidade, herbicida inibidor de EPSP sintase (5 enolpiruvilchiquimato-3-fosfato sintase). Segundo Kruse et al. (2002) um herbicida que atua em pós-emergência, considerado não seletivo, de amplo espectro de controle e que bloqueia a enzima EPSPs ocorre também o bloqueio de três aminoácidos aromáticos: triptofano, a fenilalamina e a tirosina. Moraes (1980) realizou dois experimentos em Belém do Pará e observou que o melhor resultado do ponto de vista da eficiência de tratamentos em diferentes doses e modo de aplicação do controle de capim-gengibre em competição com a seringueira foi obtido com uma só aplicação de glyphosate a 3L. ha-1 (ingrediente ativo 1500 $\mathrm{g} \mathrm{ha}^{-1}$ e $1080 \mathrm{~g} \mathrm{~L}^{-1}$ de equivalente ácido) conseguindose a erradicação total quando verificado aos 60 dias e ausência de rebrotas aos 120 dias. Entretanto, precisase observar a seletividade do produto em questão em função de apresentar espectro de controle muito amplo e praticamente não há seletividade. A translocação é melhor em plantas com alta atividade metabólica (Silva et al., 2014). Nos outros períodos observados este tratamento obteve resultado de controle acima da testemunha.

A associação MSMA + diuron aos 7 e 21 dias com diferença significativa pelo Teste de
Tukey à $5 \%$ de probabilidade, o MSMA o sitio de atuação não é totalmente conhecido, mas absorvido essencialmente via foliar em contato, por isso usado em pós-emergência. Aos 28 a 63 dias após aplicação (DAA) o resultado do tratamento foi semelhante à testemunha.

Moraes (1980), comenta sobre a rapidez do MSMA em controlar a área foliar do capim-gengibre com 7 dias após aplicado, o mesmo acontece com 0 Paraquat, ambos de ação de contato evidenciando os resultados obtidos. O resultado da associação MSMA + diuron e o paraquat + diuron foram semelhantes nesta avaliação.

Dos 28 aos 63 dias destacou-se como mais eficiente metsulfuron methyl. Scholoss (1990); Durner et al. (1991) relatam o mecanismo de inibição da ALS (enzima aceto-lactato-sintese), enzima chave na rota de biossíntese de aminoácidos: valina, leucina e isoleucina. Bianchi (2014) cita que plantas sensíveis aos herbicidas inibidores da enzima ALS tem seu crescimento retardado ou inibido rapidamente (poucas horas), porém os sintomas físicos podem levar alguns dias para aparecer e a morte das plantas levar algumas semanas. Ferreira et al. (2008) citam que a ação do produto inibidores de ALS nas plantas daninhas sensiveis pode ser observada através de clorose das 
folhas e morte das gemas apicais, com evolução para morte das plantas até 21 dias após aplicação.

As principais características morfológicas do capim-gengibre que dificultam o seu controle químico é como plantas estoloníferas e rizomatosas, de porte ereto ou semi-ereto. Apresenta sistema subterrâneo com rizomas longos e rígidos, revestidos por curtas escamas, desenvolvendo-se horizontalmente logo abaixo da superfície do solo. Cunha (1971) cita a importância de obter um herbicida sistêmico no controle do capimgengibre. A dificuldade nesse tipo de manejo na cultura canavieira consta na necessidade de herbicida em ação sistêmica em pós-emergência e seletivos a cultura. Moraes (1980) observa não ser interessante a associação de herbicida de contato com sistêmico, pois o herbicida de contato anula a atuação do outro.

O comportamento explicado na Tabela anterior também foi visto nos modelos apresentados na Figura 1. Os modelos correspondem estimativas, ao modo útil para indicar a tendência dos dados. Os modelos obtidos são explicados pela equação polinomial quadrática com bons coeficientes de determinação $\left(R^{2}\right)$.

Figura 1. Controle visual (\%) do capim-gengibre com herbicidas ao longo do tempo em ensaio de campo, Rio Largo - AL, 2013.

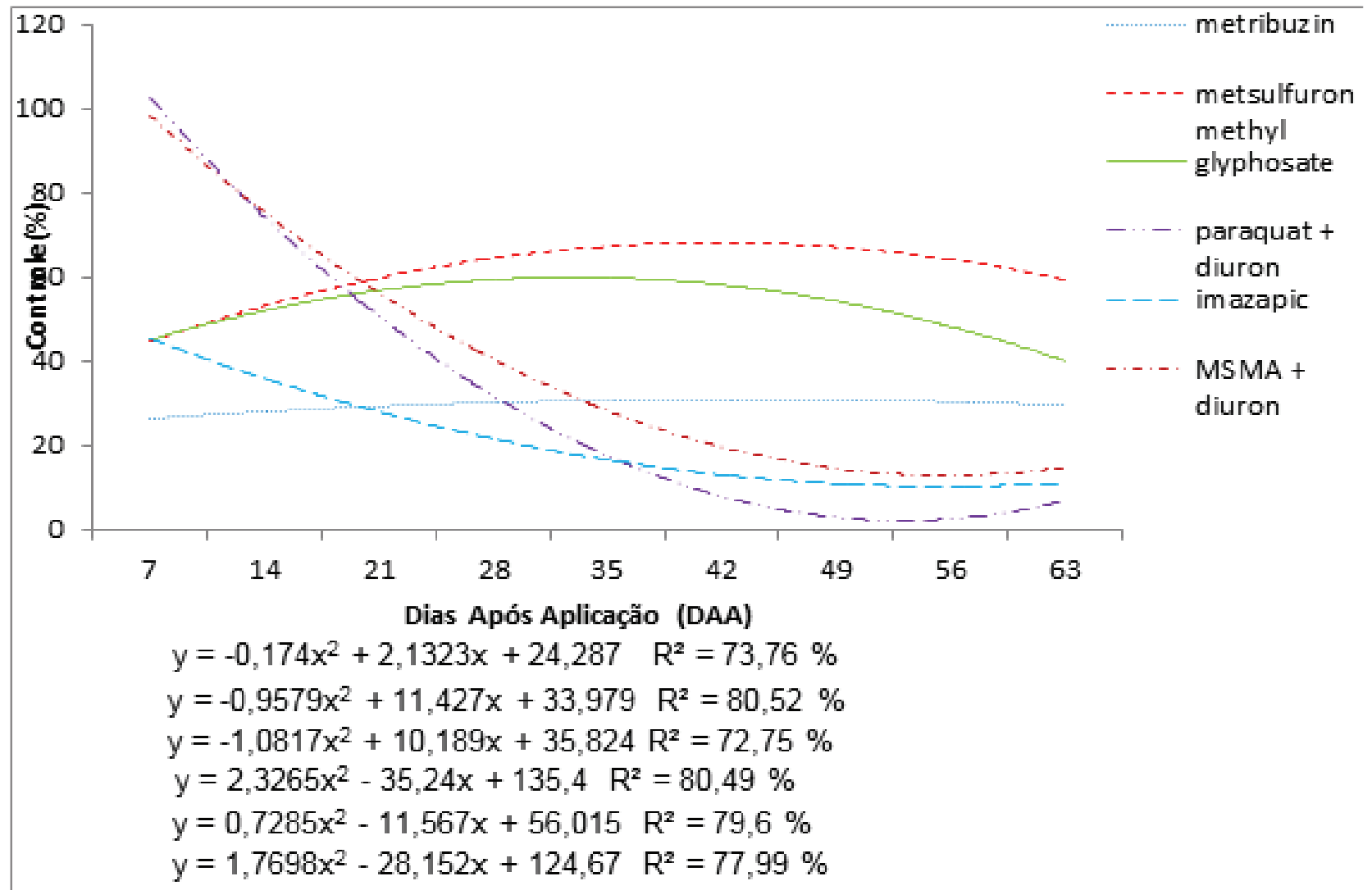

Os resultados permitiram concluir que o controle de capim-gengibre foi diferenciado pelo tempo. Aos 7 e 21 dias após aplicação para associações MSMA + diuron e Paraquat + diuron. Aos 28 a 63 dias após aplicação para metsulfuron methyl e aos 28 a 49 dias após aplicação para glyphosate.

\section{REFERÊNCIAS BIBLIOGRÁFICAS}

Balke, N.E. Herbicides effects on membrane functions. In: DUKE, S.O. Weed Physiology. Boca Raton, EUA: CRC Press., 1985. 257 p.
Bianchi, M.A. Sintomas e danos de metsulfuron-methyl em soja. Informativo Fundacep, 2009. Cruz Alta - RS, v. XVI, p. 1-4.

Cunha, H.M.P. Ação de herbicidas no combate ao capim-gengibre Paspalum maritimum Trind, em pomar cítrico. 1971. Cruz das Almas - BA: IPEAL, 3p.

Durner, J.; Gailus, V.; Böger, P. New aspects on inibitions of plant acetolactate synthese dependo on flavin adenine dinucleotide. Plant Physiology, 1991, 95, 1144-1149. 
Ferreira, A.F.; Silva, A.A.; Galon, L.; Concenço, G.; Ferreira, E.A. Mecanismos de ação de herbicidas In: Zambolim, L.; Pincanço, M.C.; Silva, A.A.; Ferreira, L.R.; Ferreira, F.A.; Jesus Junior, W.C. Produtos Fitossanitários (Fungicidas, Inseticidas, Acaricidas e Herbicidas). Viçosa-MG: UFV/DFP. 2008, 349- 393.

Fontes, H.R. Práticas de manejo de entrelinhas recomendadas para revitalização das áreas cultivadas com coqueiro da variedade gigante. 2010. Disponível em: www.diadecampo.com.br/Zpublicher. Acesso em 11/03/2014.

Kruse, N.D.; TREZZI, M.M.; VIDAL, R.A. Herbicidas inibidores da EPSPS revisão de literatura. Revista Brasileira de Herbicida, 2000, 1, 139-146.

Maciel, J.R.; Oliveira, R.C.; Alves, M. Aspectos da distribuição e conservação do Paspalum L. (Poaceae) em Pernambuco. Hoehnea, 2010, 37, 801-812.

Martins, T. Herbicida Paraquat: conceitos, modo de ação e doenças relacionadas. Semina: Ciências Biológicas e da Saúde, 2013, 34, 175-186.
Moraes, V.H.F. Controle do capim-gengibre na cultura da seringueira. Comunicado Técnico, n. 14, agosto/1980, Manaus- AM: Embrapa. 3p.

Oliveira, R.C.; Santana, S.H.; Silva, A.S.; Maciel, J.R.; Valls, J.F.M. Paspalum (Poaceae) no Rio Grande do Norte, Brasil. Revista do Jardim Botânico do Rio Janeiro, 2013, 64, 847-863.

Scholoss, J.V. Acetolactate synthase, mecanism of action and its herbicide binding site. Journal of Pesticide Science, 1990, 29, 283-292.

Silva, A.A.; Silva, J.F; D’Antonino, L.; Ferreira, F.A. Classificação e mecanismos de ação de herbicidas. In: Zambolim, L.; Silva, A.A.; Picanço, M.C. O que engenheiros Agrônomos devem saber para orientar 0 uso de Produtos Fitossanitários. Viçosa- MG: Os Editores, 2014, 115-152. 